\title{
Factors promoting shared decision-making in renal replacement therapy for patients with end-stage kidney disease: systematic review and qualitative meta-synthesis
}

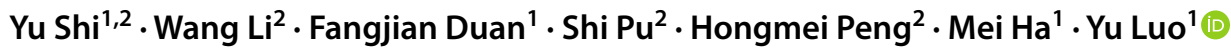

Received: 18 March 2021 / Accepted: 6 June 2021 / Published online: 22 June 2021

(c) The Author(s) 2021

\begin{abstract}
Purpose Shared decision-making (SDM) about the type of renal replacement therapy to use is a matter of great importance involving patients, their families, and health treatment teams. This review aims to synthesize the volume of qualitative work explaining the factors influencing SDM regarding renal replacement therapy.

Methods A systematic review and qualitative meta-synthesis approach recommended by JBI was used, six databases were searched. Studies were qualitative or mixed research published since 2000, with a primary focus on patient experiences, perceptions and practices regarding which method to choose for renal replacement therapy in End-Stage Kidney Disease (ESKD) patients. All themes were analyzed and compared to the established connectedness.

Results A total of 1313 patients were enrolled in 32 studies focusing on factors associated with SDM regarding renal replacement therapy were included. All quality evaluations of the literature were medium to high. Four common themes were identified in our synthesis: (1) patient personal reasons, (2) family-related factors, (3) health care professional-related factors, and (4) social factors influence.

Conclusion The model proposes pathways that could be explored further in future qualitative and quantitative studies and suggests that patients' beliefs, emotions, and awareness should be targeted alongside patients' decision-making practices to increase the efficacy of interventions. The majority of studies included in this review focus on older patients, and all report patients' perspectives. Further research is required to understand the family member perspectives on SMD of renal replacement therapy.
\end{abstract}

Keywords ESKD $\cdot$ Renal replacement therapy $\cdot$ Decision-making $\cdot$ Qualitative evidence synthesis $\cdot$ Systematic review

Yu Luo

luoyuhlgl@tmmu.edu.cn

Yu Shi

18580642512@163.com

Wang $\mathrm{Li}$

18929268893@163.com

Fangjian Duan

13108978393@163.com

Shi Pu

260187135@qq.com

Hongmei Peng

wavvmm1212@sina.com
Mei Ha

976506580@qq.com

1 School of Nursing, Third Military Medical University (Army Medical University), No. 30 Gaotanyan Street, Shapingba District, Chongqing 400038, People's Republic of China

2 Department of Nephrology, The Key Laboratory for the Prevention and Treatment of Chronic Kidney Disease of Chongqing, Chongqing Clinical Research Center of Kidney and Urology Diseases, Xinqiao Hospital, Army Medical University (Third Military Medical University), Chongqing 400037, People's Republic of China 


\section{Background}

Chronic kidney disease (CKD) has become a global public health problem with occurrence steadily increasing by approximately $6 \%$ annually, the global all-age mortality rate from CKD increased $41.5 \%$ between 1990 and 2017 [1, 2]. End-stage kidney disease (ESKD) treated by renal replacement therapy: dialysis and kidney transplantation increasing by $43.1 \%$ and $34.4 \%$ [3].Generally, patients with ESKD represent approximately $0.03 \%$ of the total population in many high-income countries, but their treatment alone accounts for approximately $2-3 \%$ of the annual healthcare budget [4]. After entering ESKD, patients are faced with a choice of three alternative treatment schemes, including hemodialysis, peritoneal dialysis or kidney transplantation, and it is not easy to identify the most appropriate treatment scheme in the face of different settings. Clinical guidelines point out that advanced CKD patients who need renal replacement therapy should be offered different dialysis modalities and be provided different dialysis options, timely education, and support them to choose the most needed and value-oriented methods [5]. (e.g., hemodialysis vs peritoneal dialysis), there are a number of other decisions made throughout the CKD trajectory, including those related to lifestyle and diet, medication, and advance care planning [6].

Therefore, it is necessary to invoke a doctor-patient shared decision to make a choice. Shared decision-making (SDM) interventions broaden patients' knowledge sources and decrease doctor-patient conflict, promoting decisionmaking about care and treatment based on informed preferences [7]. SDM means that patients' options are well informed to them and available information could be well considered by them, in that case, decisions are customized to the values and preferences of each patient. Despite SDM emerging as a pillar of national and international quality standards and policies, evidence showed that CKD patients have limited participate in SDM [8]. Regarding SDM, several aspects might jeopardize information transfer [9-12]. First, the accurate information on the expected outcomes of different options may not simply be available or just do not available to some specific group where the patient belongs (generalizability, external validity). Second, such information can be transferred in a biased, non-neutral way. Third, the information is often transferred in a way that could confuse the patient. Fourth, information on outcomes that matters to the patient may not be available, whereas ample information providing on outcomes does not matter to the patient.

Patient decision-making in the management of ESKD has reached increasing attentions. Doctor-patient SDM on the choice of renal replacement therapy is not only reflected in the patient's decision factors but also includes family factors, social factors, and very critical health care factors because much of the decision-making information comes from professional health care teams. There have relevant studies on the integration of viewpoints when patients with ESKD enter the choice of renal replacement therapy [13, 14], including (1) patient's personal reasons, (2) family-related factors, (3) health care professionalsrelated factors, and (4) social factors. And experiments in the field of SDM on dialysis modality highlight that patients tended to be strongly influenced by the stories of other patients and had far less by the same information provided by a physician [15]. Therefore, the purpose of this review is to examine the patterns and themes of modality decision-making and synthesize these findings into more generalized knowledge claims regarding by meta-ethnography, so as to find out which factors affect the decision-making of ESKD patients mostly and provide an evidence-based foundation for the formulation of doctor-patient SDM assistance schemes in CKD management.

\section{Methods}

\section{Research design}

A meta-synthesis approach (following JBI guidelines [16, 17]) was used to synthesize the qualitative literature on patient decision-making regarding renal replacement therapy. This approach is grounded in pragmatism and phenomenology to assist synthesis of qualitative studies [18]. Meta-synthesis following JBI guidelines [16] is detailed below (and in SI-1).This research work was carried out between March 2020 and January 2021.

\section{Search strategy}

Search using all identified keywords and index terms was undertaken across 9 Chinese and English databases, including PubMed, CINAHL, Embase, Cochrane Library, CNKI, Sinomed, Wanfangand VIP. Collected literatures on decision-making in patients with ESKD from January 2000 to January 2021 (Although research on SDM in patients with ESKD began to appear in 1996, we considered the research at that time to be too old), and the references included in the study were screened. Combination of subject words and free words, and searched all synonyms as far as possible, the search words include End-Stage Kidney Disease/Renal Kidney Replacement Therapy/Shared decision-making/Qualitative research, etc. (in SI-2). 


\section{Inclusion criteria}

\section{Types of participants}

This review considered studies focusing on views and experiences of patients or family members or healthcare professionals (e.g. physicians and/or nurses and/or pharmacists) in decision-making about the treatment of ESKD (defined as using qualitative techniques for recruitment strategies, data collection, and data analysis) (in SI-3).

\section{Phenomena of interest}

The phenomena of interest for this review were real experience, perceptions and feeling of ESKD patients or family members or healthcare professionals in the SDM of renal replacement therapy.

\section{Context}

Diagnosis of ESKD, treatment decision RRT and the whole process after selection. These deployment settings were in any country, cultural context or geographical location.

\section{Types of studies}

Different types of qualitative research, including designs based on phenomenology, ethnography, grounded theory, action research, field research, etc.

\section{Assessment of methodological quality}

Qualitative papers selected for retrieval were assessed by two independent reviewers for methodological validity prior to inclusion in the review using JBI Qualitative Critical Appraisal Checklist [16] (reported in Table 1). The evaluation contents were 10 items, and each item was evaluated as "Yes", "No", "Not clear" and "Not applicable". The research quality was divided into A, B and C, 3 grades. Quality levels that meet all criteria are $\mathrm{A}$, those that partially meet $\mathrm{B}$, and those that do not meet $\mathrm{C}$ at all. All disagreements between the reviewers were resolved after discussing with a third reviewer.

\section{Data extraction and synthesis}

Literature screening and data extraction were conducted independently by two researchers, and mutual verification was conducted. If there were any disagreement, the third researcher shall make the ruling if it cannot be determined after negotiation between the two parties. Through repeated reading, full understanding and reasonable interpretation of the included original research, we grasped the relationship between different research results, interpreted the meaning of different research results, combined similar themes to form a new category, and summarized the new category into integrated results to obtain a new interpretation of the phenomenon. Full texts were imported to NVivo qualitative data analysis software, QSR International Pty Ltd. Version 12 for Qualitative Research. General details of papers were composed of author, published year, methodology, data analysis, setting of the research, demographics of participants, data analysis and conclusion (Table 1).

\section{Results}

\section{Study characteristics and assessment of methodological quality}

As shown in Fig. 1, thirty-two studies were included in the final review. The characteristics of these studies are listed in Table 1. Studies represented a total of 1300 ESKD patients (with or without RRT). Two of the included studies (Study 2 $\&$ Study 18) were mixed-method studies, and in these cases, only qualitative findings were included.

\section{Three-dimensional integration of studies (building a whole pattern from the individual parts)}

By synthesizing the results of these 32 studies, we produced 334 codes. In stage 6 of the analysis, the first reviewer combined similar metaphors to produce 139 s-order constructures. The third constructure is further grouped into four themes [13, 14]: (i.) patients' personal reasons influence treatment decision-making; (ii.) family-related factors influence patients' decision-making; (iii.) health care professionals-related factors influence patients' decision-making; and (iv.) social factors influence patients' decision-making). The outcome of these themes explains the meaning of these constructures and how they relate to each other, and the final themes and subthemes form a three-dimensional integrated model, as shown in the conceptual model in Fig. 2.

\section{Theme 1: Patients' personal reasons influence treatment decision-making}

\section{Personal values to RRT}

It is common to see that before patients make treatment choices, they would explicitly express how they are weighing the benefits and disadvantages of RRT. In other words, will this choice allow them to continue their current lifestyle, or/or may allow them to have more flexibility in their lifestyle? Studies have indicated that a significant group of 


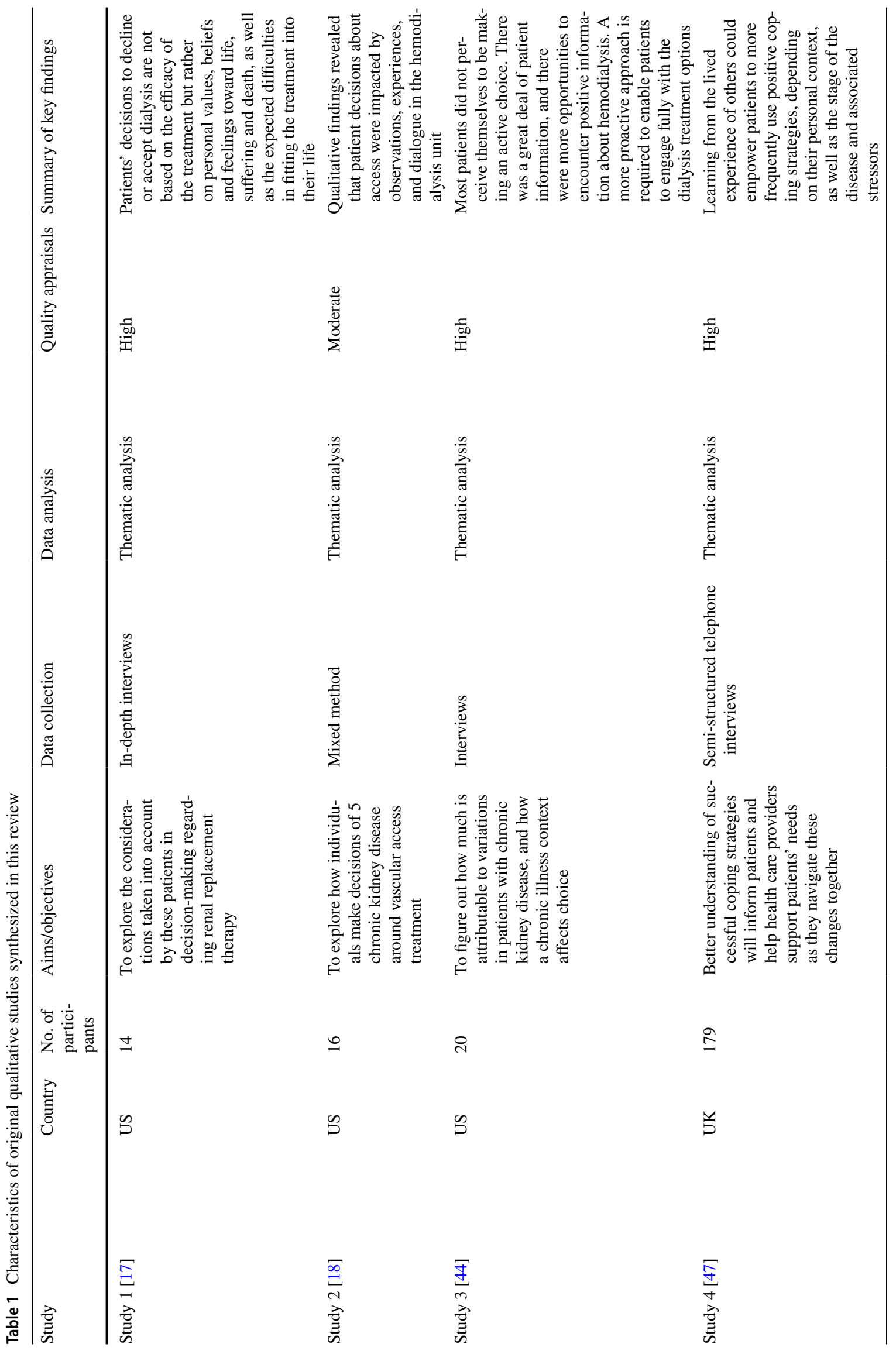




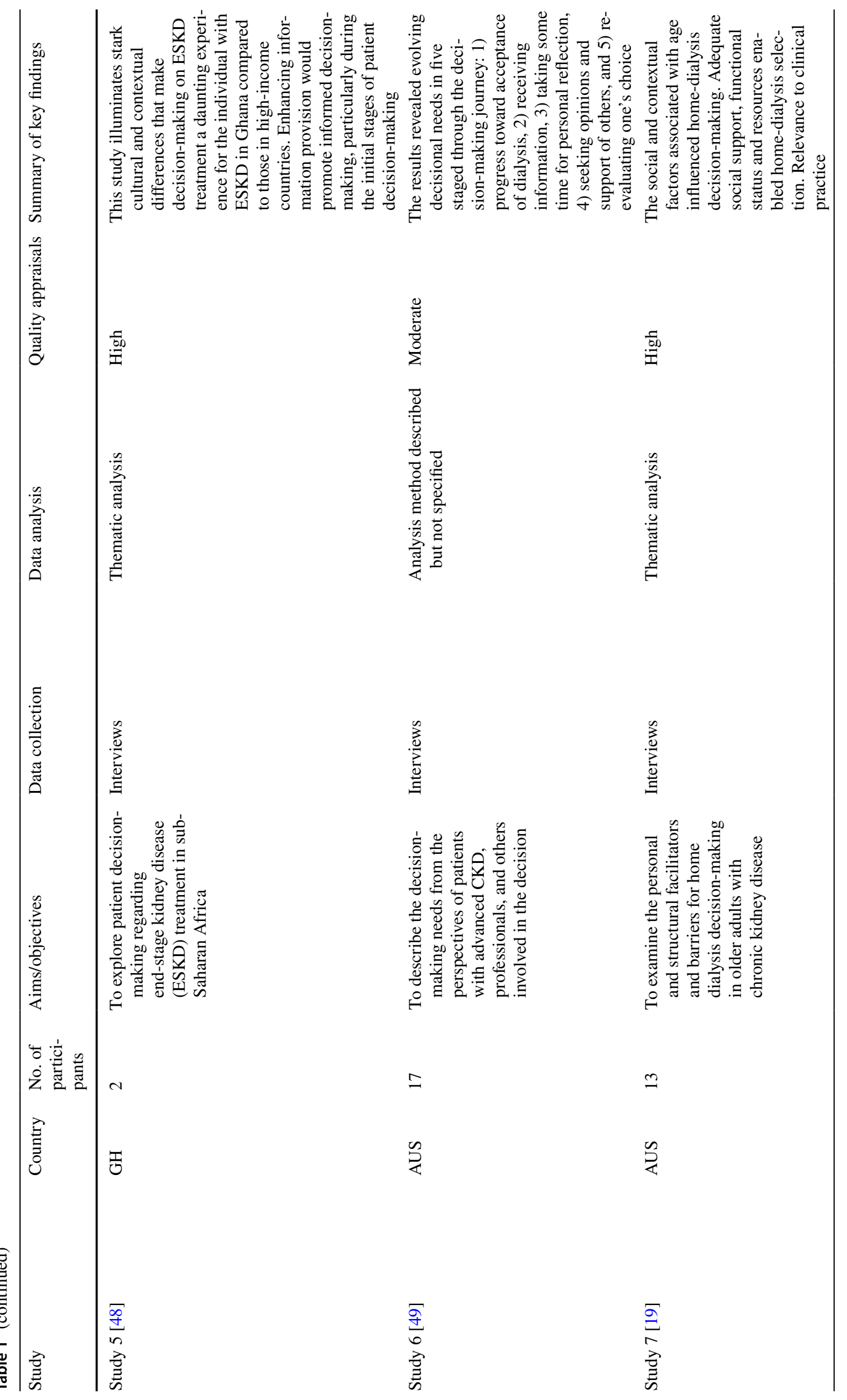




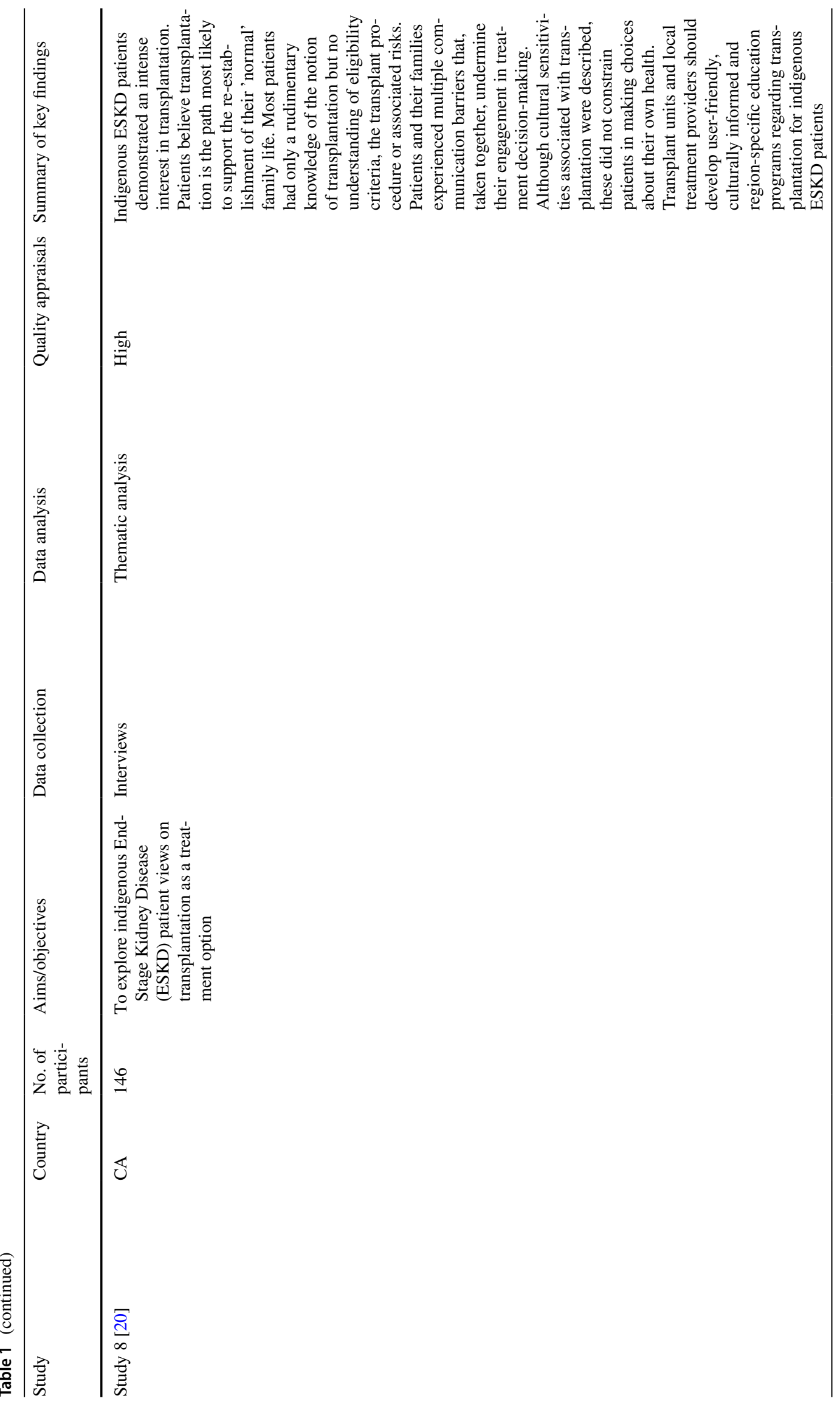

黑 Springer 


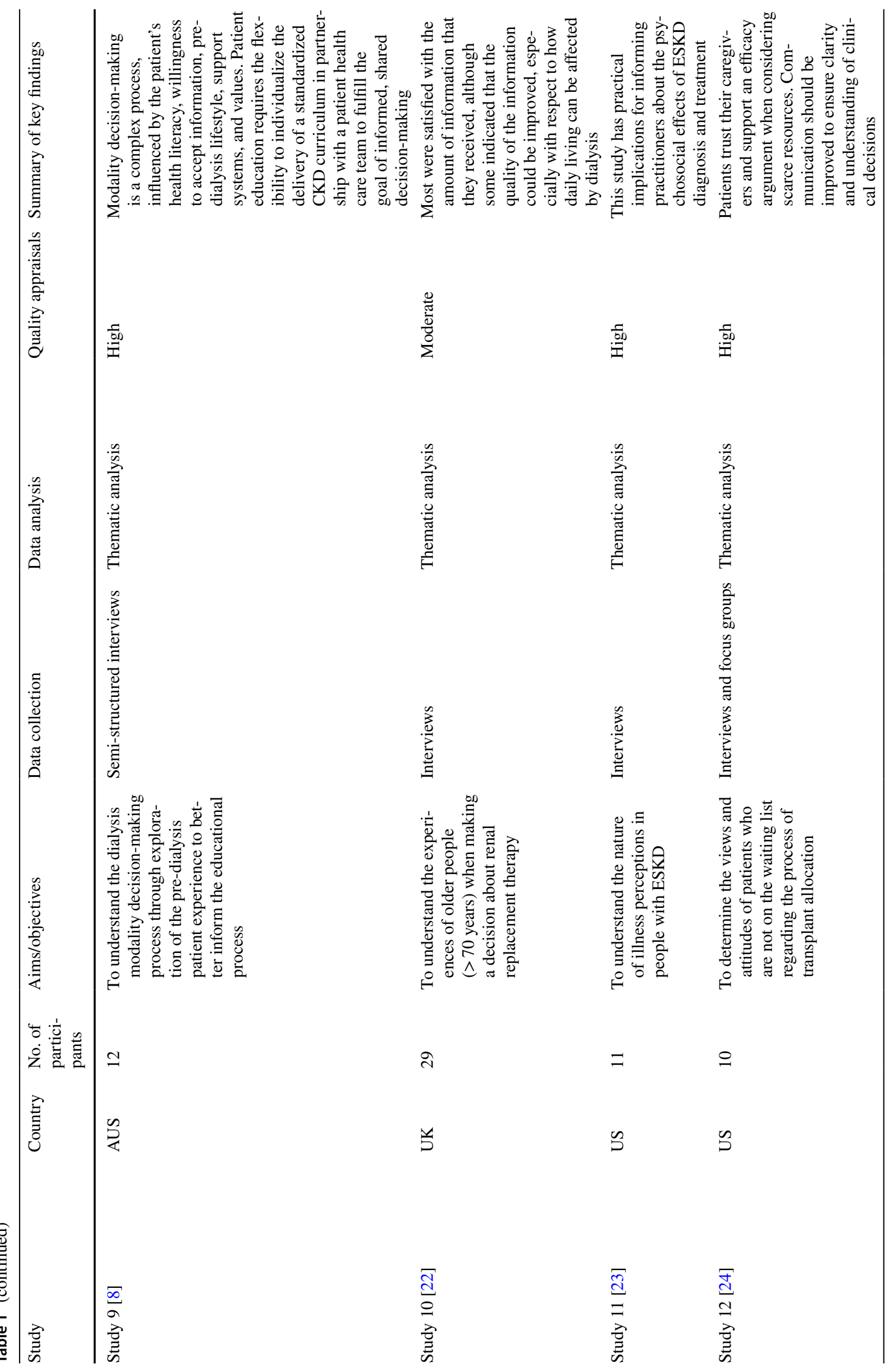




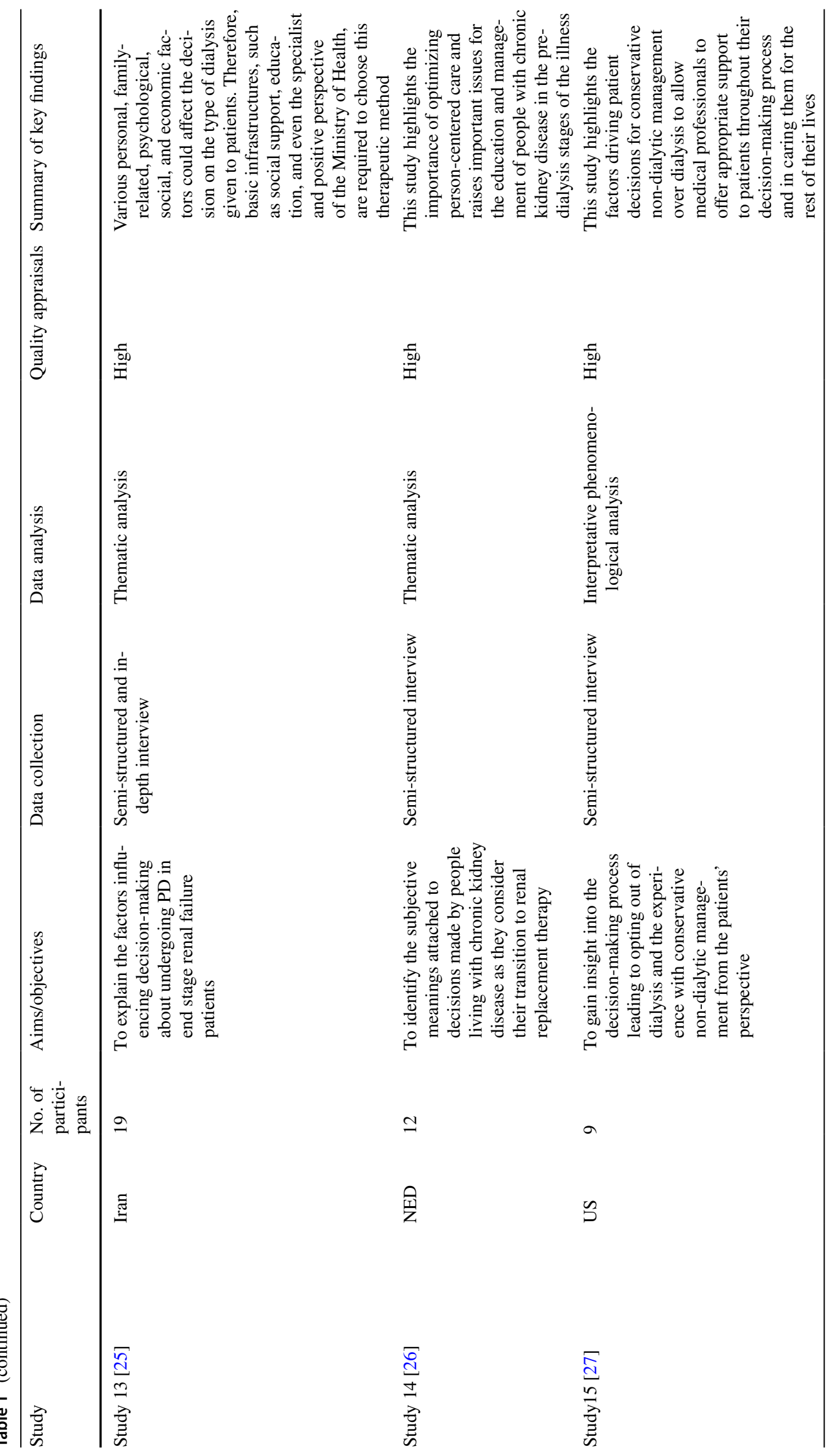




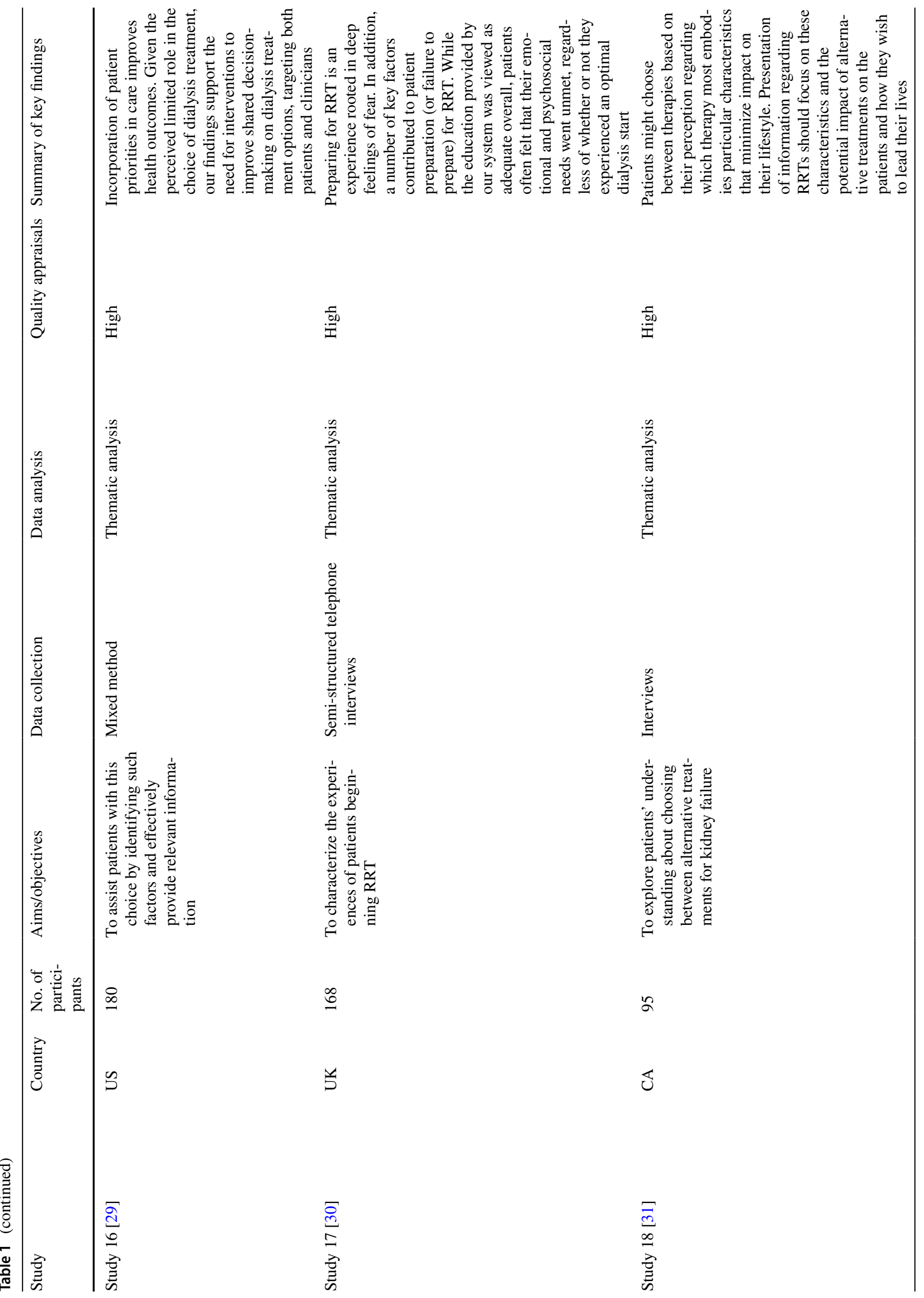




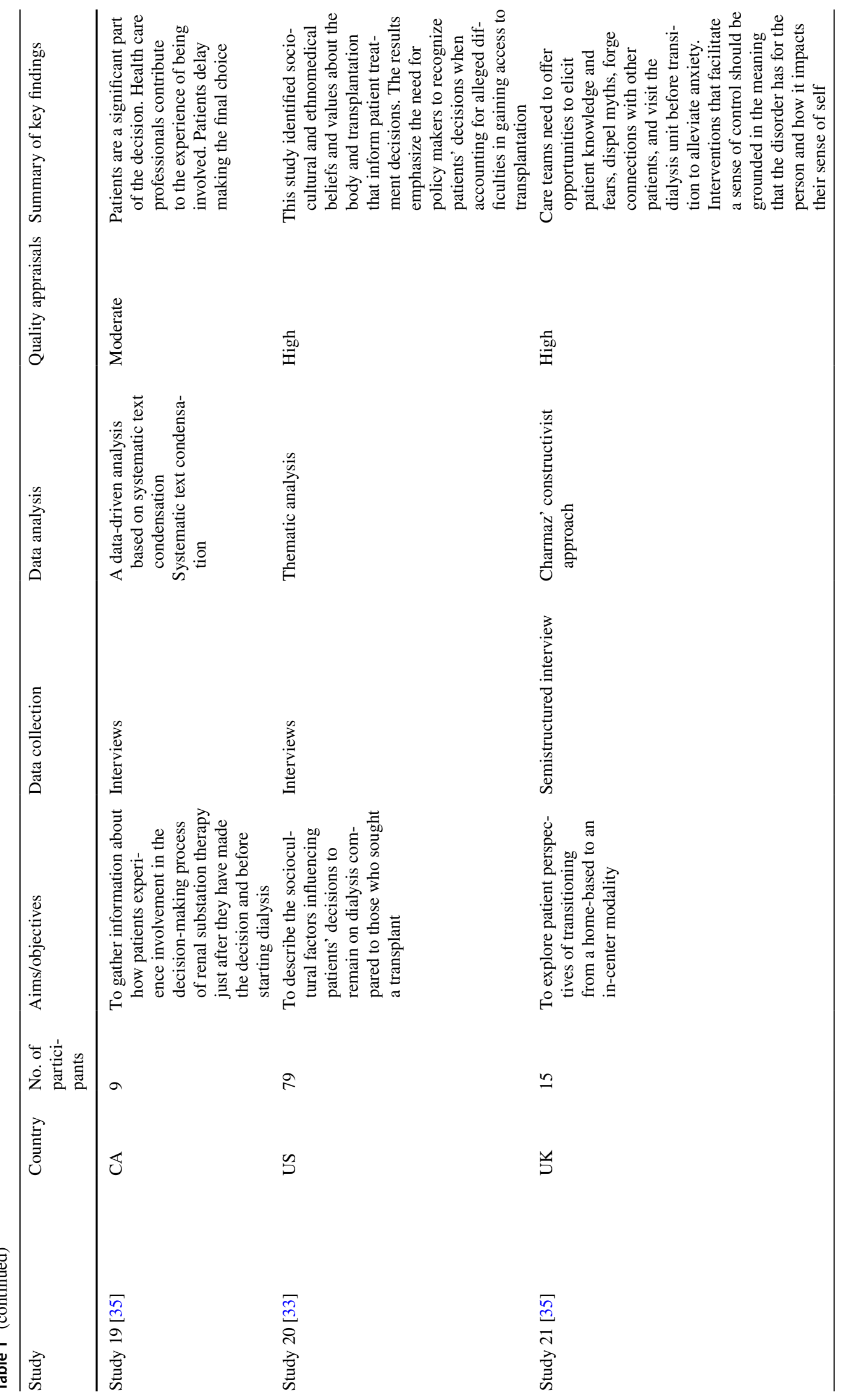


International Urology and Nephology (2022) 54:553-574

563

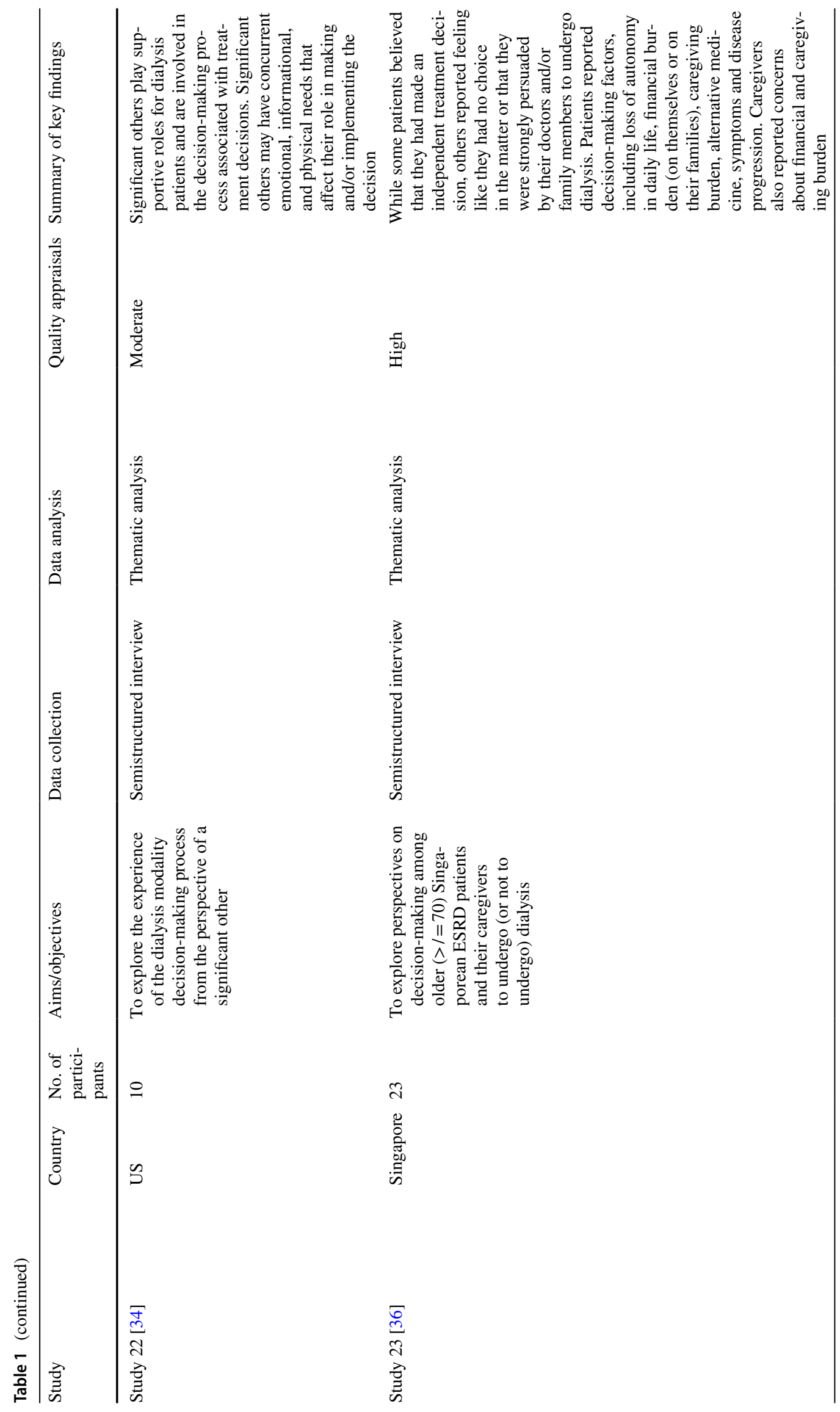

Springer 


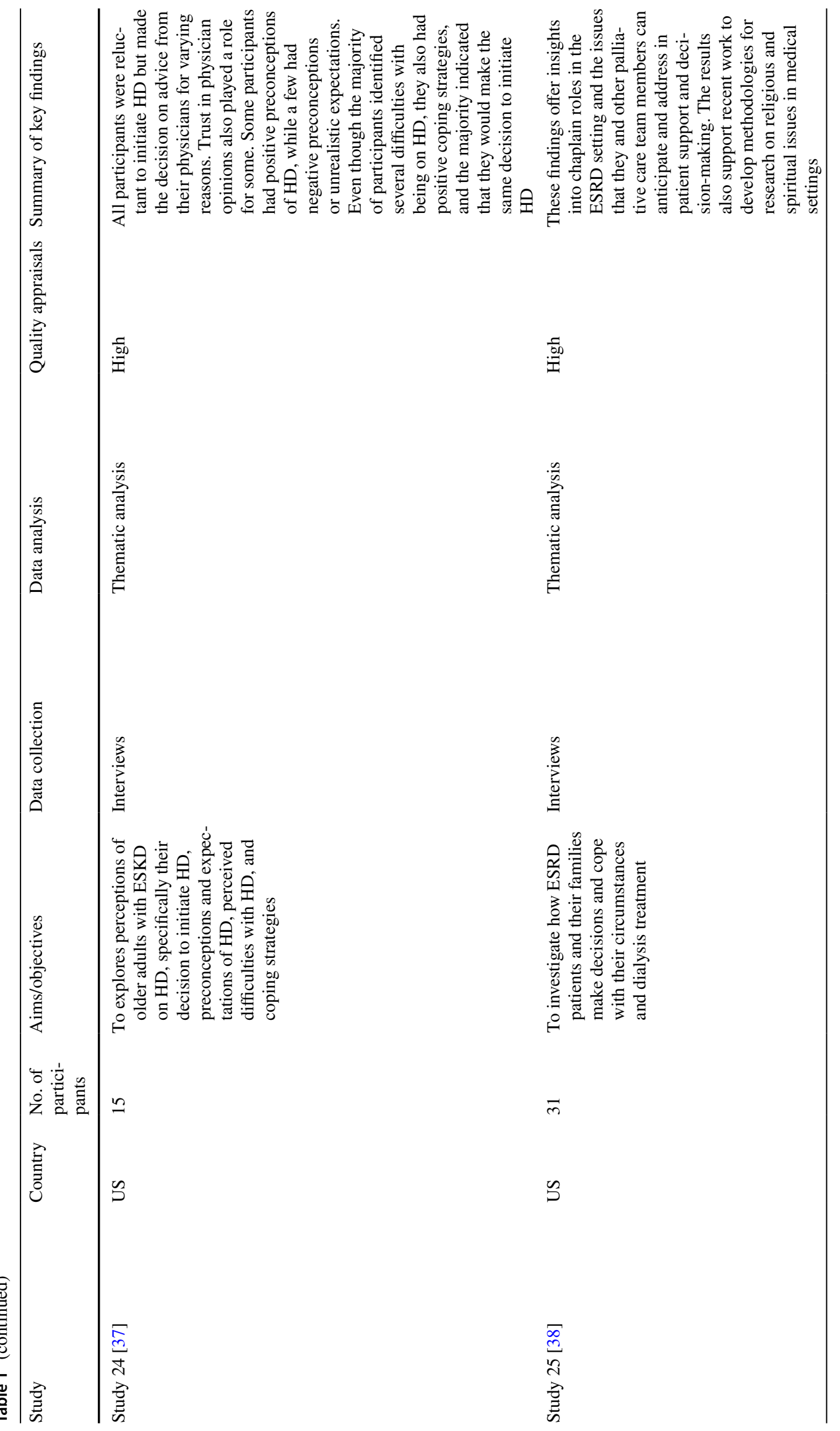




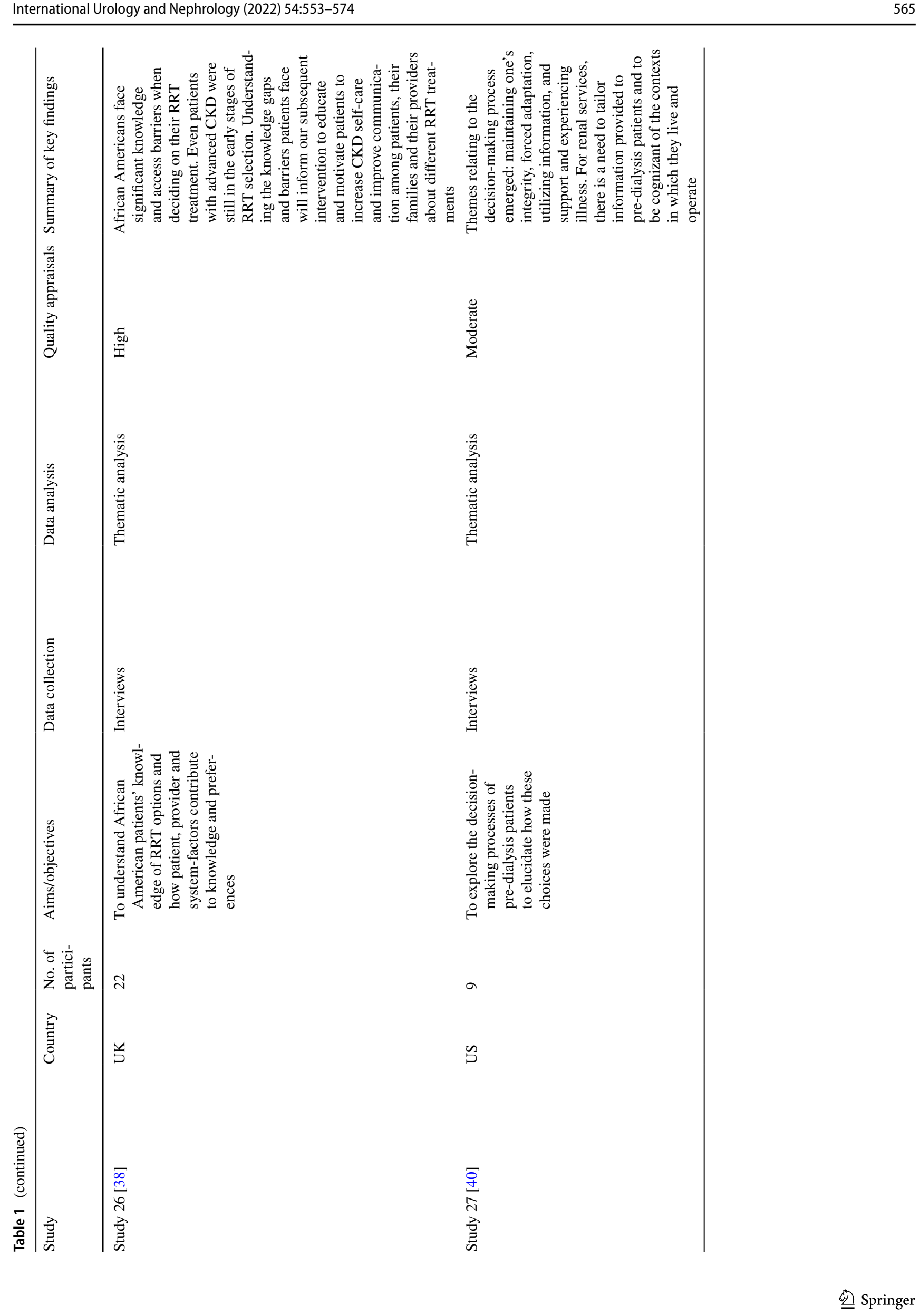




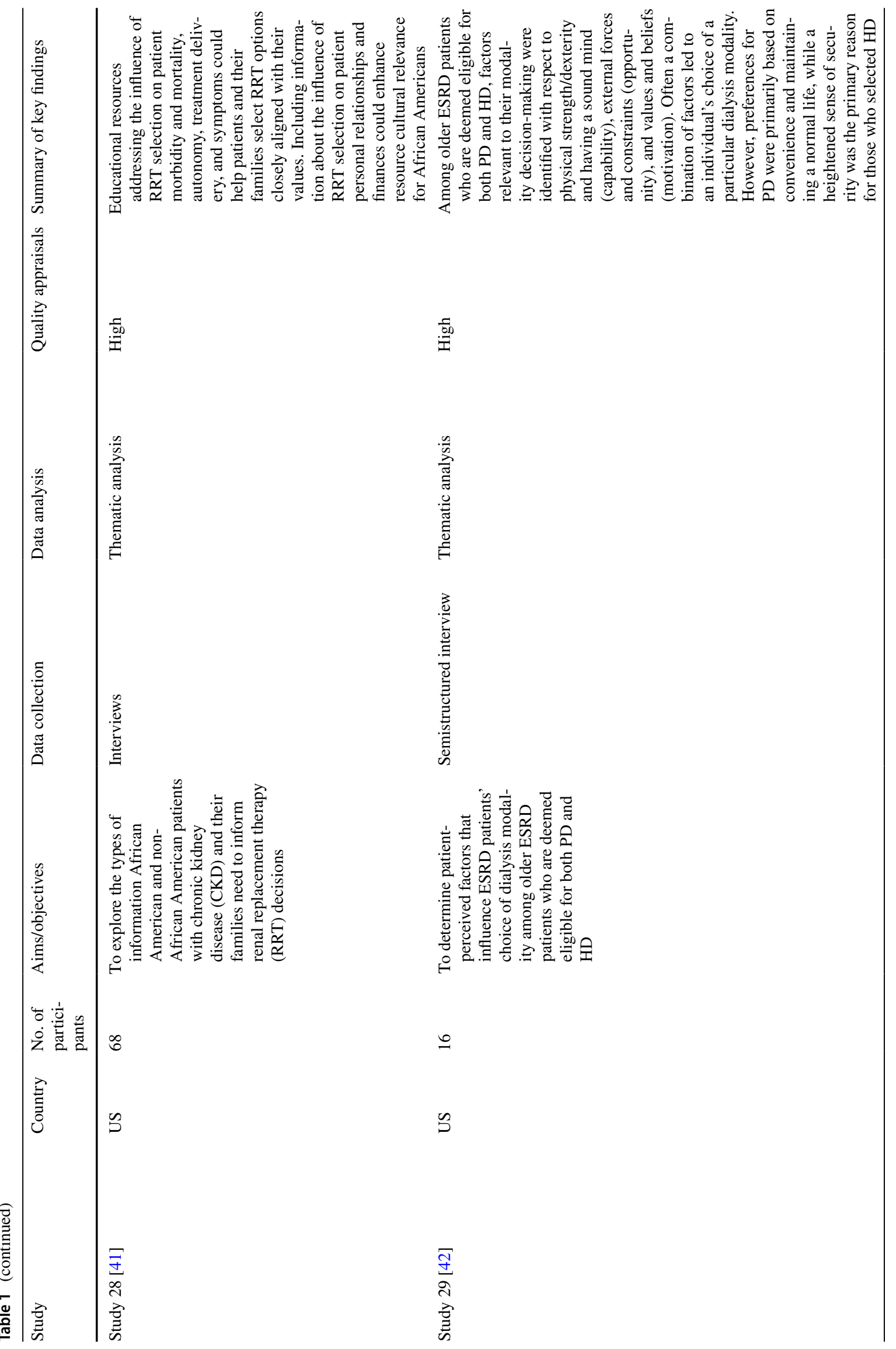




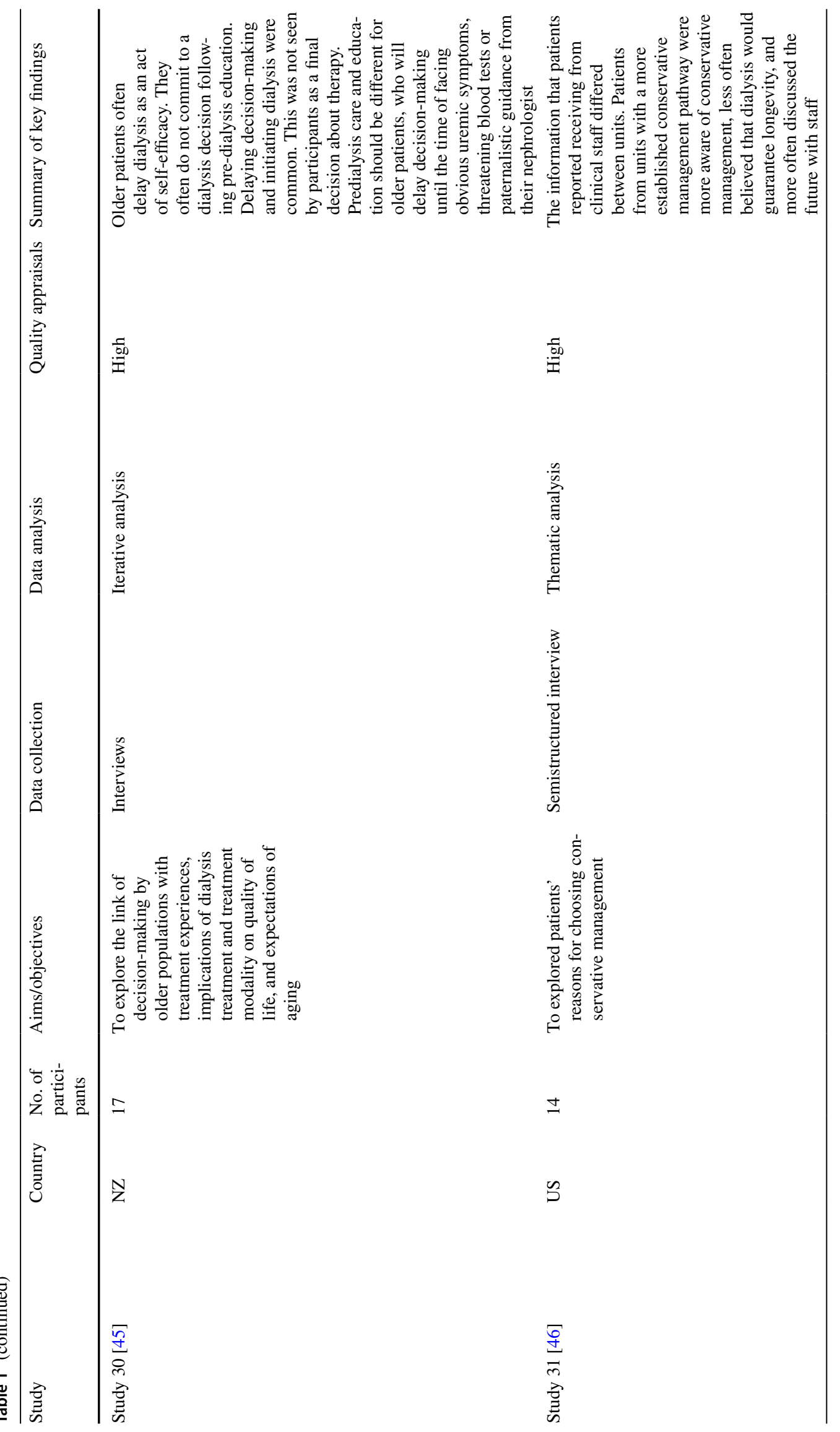




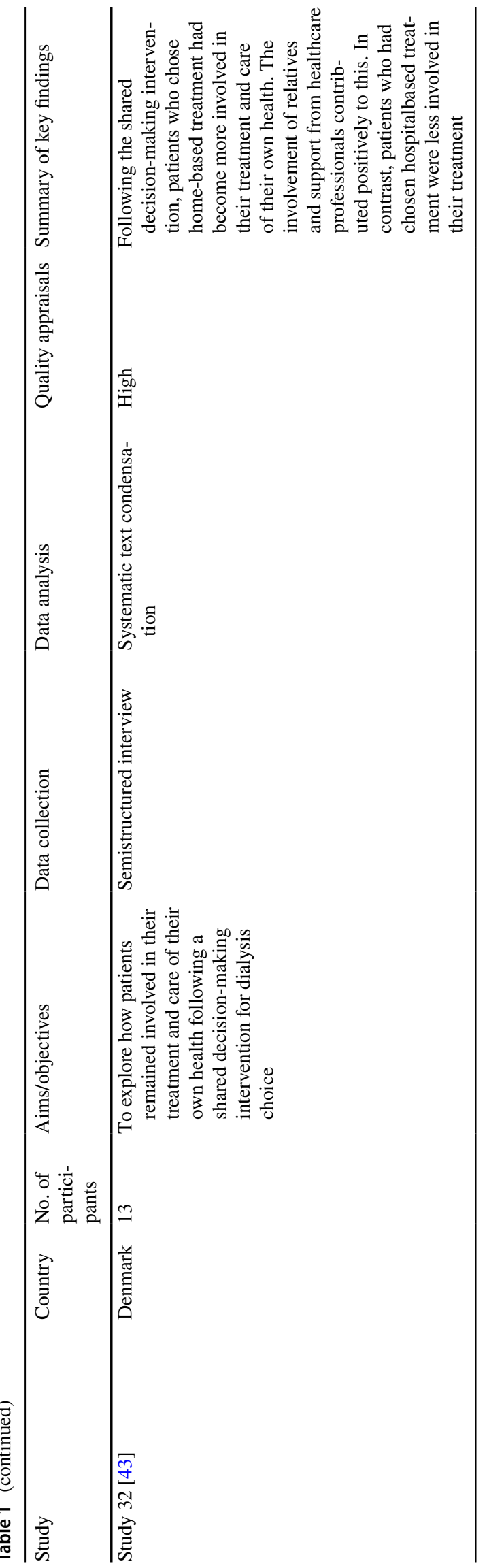

patients' value orientation to RRT makes influences to their treatment options.

One of the main reasons why patients refuse dialysis treatment is that they perceive it will deprive them of the right to make their own choices. To them, dialysis will cause more problems, forcing them to give up their freedom and become dependent on medical treatment. In order to be free, they would rather forgo a longer life than to be restricted by a comprehensive treatment such as dialysis [19, 20]. (studies $1,9,18)$.

“... my lifestyle, I am very independent and really didn't want to be tied to a clinic."

"I am now 77, and you can twist it one way or the other, dialysis is a trouble to go through, it is just a way to postpone death. I don't see it happening to go to the hospital 3 times per week, I am already too weak, in particular physically. I am worn out!"

Participants often complained that dialysis treatment wasted their time, not only the treatment itself, the travel to and from the hospital, the waiting and the recovery from the treatment are all added their time costs. They acknowledge that their lives have been changed and it may also continue to have impacts on the future lives [21-24]. (studies 14, 16, 17, 24, 26).

For patients, another key factor in the process of making and implementing decisions is the impact those decisions have on their personal health [25-28]. (studies 7, 15, 21, 22, 32 ). There are also studies showing that patients' incorrect perception of RRT leads to patient selection [29] (study 5), or patients feel that because of their disease symptoms, they have no choice but to choose dialysis [22, 30-32] (studies $17,20,25,27)$.

"I knew that when [I] come to dialysis for, let's say 10 times or 6 times, it will go. So I was coming here happy since I was going to be $\mathrm{OK}$. ”

\section{Strategies and behaviors for dealing with RRT}

During the beginning of new activities, the participants initiated some primary changes. This developed from the positive changes in outlook experienced, for example, see previous works [30-34]. Participants would use the time spent on RRTs to do something constructive (studies 4, 6, 17, 20, 25): participate in social activities (such as grocery shopping), get close to nature ("The doctor told me it was the best thing I could do. Get out and walk.”), learn a new musical instrument ("I play guitar for the church and I got back to that"), perform charity activities ("I talk to them [other patients with ESKD], sit with them, I explain things to them. In fact, I even support a few of them if they are acutely financially distressed"). 
Fig. 1 Flow chart illustrating selection of studies through database searches, screening, team discussions and supplementary searches
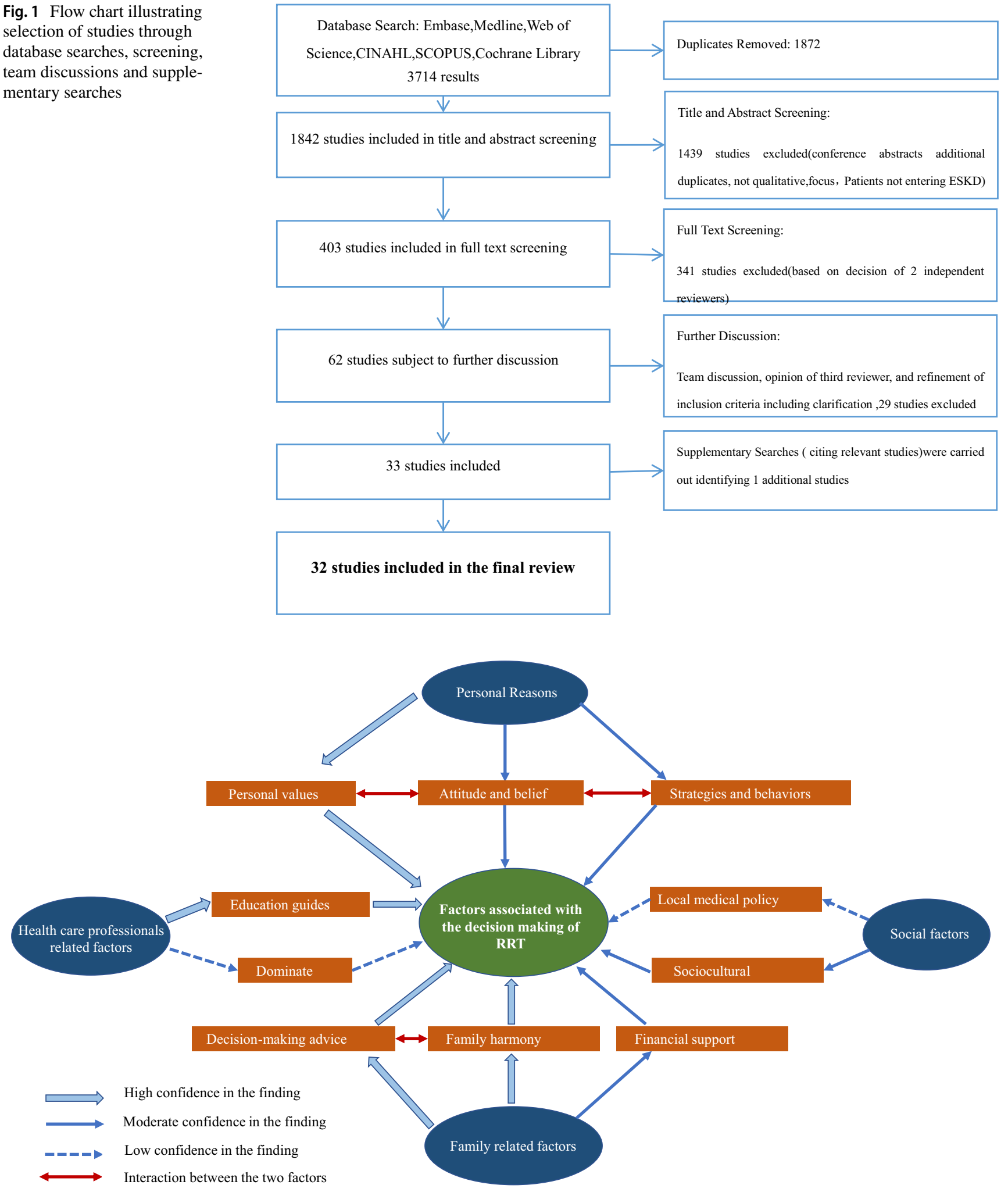

Fig. 2 Conceptual model illustrating 4 constructs 


\section{Attitude and beliefs to cope with RRT}

Patients may also feel fear, denial, regret, anger and shock due to the lack of understanding of their current health condition. Negative emotions, such as fear and ambivalence, often occurred during the preparation period, regardless of whether the patient is in optimal or suboptimal condition at the beginning of RRT. As described by patients, if situation allows, they would like to put off preparatory surgery of dialysis access for as long as possible, and they did not deny that they were willing to delay the dialysis initiation in this way. Numerous factors [22, 31, 32, 34-36] contributed to this sense of fear, including concerns about the AVF placement procedure and/or dialysis, that dialysis may eventually tend to be a death sentence, and lifestyle changes due to the requirements of RRT (studies 5, 6, 12, 14, 15, 25, 27). Despite the difficulties and limitations, most patients are still willing to take whatever steps they can to prolong their lives. "We all die at some point, and we all know that. Yet, somehow it never dawned on me. It is almost as if I never realized that; I was simply living.... as if life is eternal...." Those who chose dialysis showed a desire to survive, and some said they hoped to receive a kidney transplant.. [22, 35] (studies 6, 14). Such optimism made them more positive about the disease and treatment: "It is not all roses, but with a crisis like this I believe you have, have to stay positive and you have, have to believe in hope." The meaning of "beingwith" is to provide emotional, physical, psychological and spiritual help/support to the patient. It is also described as general and all-encompassing: "My part is just to support." As another [36]patient shared, "Being there and listening and supporting the ideas" (study 17).

\section{Theme 2: Family-related factors influence patients' decision-making}

\section{Giving decision-making advice about RRT}

Family is of significance in treatment decision-making, it often plays an indispensable role in persuading patients to start dialysis. In this research, different family-based areas, including high family interactions, good structure, intellectual and cultural background, up-to-date information, and high life expectancy are all very meaningful to the process. On the other hand, inefficiency of family influences and limited knowledge in related fields may play an inhibitory role in this regard. In different studies, families assist patients with kidney disease by supporting and rehabilitating them also influence the selection of their therapeutic method. Some of the families and friends find it very hard to encounter RRT and choose conservative treatment (studies $16,19,25)$.
Studies [31, 33, 34, 37] (studies 3, 4, 5, 20) also show that the support of family members and active participation in the communications of treatment decisions can also help patients to confront the current changes and obstacles.

\section{(Family) financial factors}

When making decisions, the financial burden of dialysis has become the first concern for many patients increasingly, because they perceived those treatment expenses would be extremely burdensome for themselves or even their families. Many studies [21, 33, 34, 36] (studies 4, 5, 15, 17) indicated that it is a huge burden to the patients because they will always need financial support and companion from the family Being financially dependent on their spouse or children for daily and medical needs, the patients may consider it is unnecessary for them to suffer from these additional financial burdens due to the high cost of dialysis.

\section{Consideration of family harmony}

Some patients acknowledged that their family or friends had truly influenced on their decisions of continuing hemodialysis. Some reasons, as cited, are that they wish to see their grandchildren to be grown up or they are caregivers of their spouse or children and are the source of income to the family [22, 26, 30, 38] (studies 4, 6, 25, 31). They discussed several negative impacts they anticipated that dialysis could cause to their lifestyle, yet, for most people, the financial burden of dialysis outweighs the benefits of longer life, even though it may provide the opportunity to spend more time with the family. In some conditions, the patients could also be caregivers to their sick or disabled spouse or children, hence they choose dialysis. In addition, the main reasons for patients to start dialysis could be like, relieving from painful symptoms, making family members happy and satisfied, and prolonging their lives as long as possible., for example, "I' $m$ doing what [my family] wanted," and "because they wanted me here longer" [30] (study 25).

\section{Theme 3: Health care professionals-related factors influence patient decision-making}

\section{Education guides patients to choose treatment methods}

To provide guidance and useful information to patients and prospective dialysis patients en masse, the Renal Nursing Team organized a Renal Patient Information Day. It is the principal channel for these patients to reach information they need. It is generally agreed that this activity plays a crucial or confirmatory role in the participants' subsequent decisions. The team also manages to improve the patient's sensitivity towards their disease. While many patients were 
satisfied with the care and compassion shown during their contact with team members, some felt there was still room for improvement in their work. The patients emphasized that the team members should give them more empathy and patience, and be willing to answer any questions that patients may have about the preparation and initiation of renal replacement therapy. These studies [22-25, 39-43] (studies $3,7,8,9,13,14,17,18,32$ ) shed light on how patients can get the exact treatment. First, patients should have relevant knowledge and educational background to their disease. Patients regard these knowledge and information more as a tool to understand and prepare for dialysis, not just to help them make decisions. Follow-up with doctors and health educators to categorize a wide range of high-quality information, organizing and analyzing relevant information and data are very helpful as well.

\section{Dominate treatment plan for ESKD patients}

The relationship between the patients and their doctors is quite complicated and has impact on their treatment decision-making. These studies [20, 22] (studies 5,7) indicated that doctors dominate patient decisions. In these studies mentioned above, the relationships between many patients and their doctors may be asymmetrical to some extent, in which the doctor's suggestions and opinions held much more weight than the patients themselves. In that case, the patients felt that they were in no position to consult their doctors and ask suggestions about their disease, but more of educating them than guiding them about medical affairs.

"There is really nothing to discuss with the doctor. The doctor is wary and persuaded me to accept dialysis... all they would do is to encourage me to go on dialysis and tell me the benefits of dialysis."

Some participants have made up their mind to initiate HD because of the physicians' opinions, and they trusted them. Longer-term doctor-patient relationships and the accompanying higher trust levels typically made patients more comfortable with their decision to begin treatment.

"And, it was much easier, I think, than if you have some, you know, you're thinking, 'Oh, he's just trying to make money' or, you know, whatever. But I trusted her [my doctor]."

\section{Theme 4: Social factors influence patient decision-making}

\section{Local medical policy}

The only study [29] (study 5) in developing countries indicated that medical policies have an impact on patient treatment choices. For many patients, the location of the treatment center greatly influences (limits) their choice of treatment. In fact, for some people, the only realistic option is to move or to travel long distances across regions to get treatment for renal services. As in many high-income countries, palliative care for ESKD in Ghana is not an option that can be openly considered.

\section{Sociocultural}

These studies [20, 21, 23, 41] (studies 4, 5, 7, 13) suggest that religious beliefs are significant in helping patients and their families dealing with dialysis. Faith brings hope and fear to the patients and their families. "I have no worries about it. Not at all. Yah, if you have that [faith], there's nothing to fear. No fear of death." said a man who was anticipating his death after discontinuing dialysis. Another patient who chose to stay on dialysis had a similar response. While some participants found pleasure in the close relationship between their grandchildren, others said faith and religion played a major role in how they coped with a medical diagnosis and received hemodialysis.

\section{Discussion}

In this study, the researchers have reviewed thirty-two latest qualitative studies mainly from websites, professional magazines on SDM about the categories of renal replacement therapy and finally synthesized numbers of findings. Meta-synthesis was included in the studies[44], and secondary analysis of the data are presented in these studies. A conceptual model (Fig. 2) was produced illustrating the relationships among our main themes: (i) patients' personal reasons; (ii) family-related factors; (iii) health care professionals-related factors; and (iv) social factors. Detailed factors and explanations have been proposed in the current qualitative literature.

In theme 1, the studies indicated that bothers came from various sources with provided evidences, and stress is associated with all sorts of personal reasons, which influences treatment decision-making. Patients cannot refuse to deal with kidney failure and its impacts on their lives and lifestyles over years and years. For example, diet and fluid restrictions, mental burdens and social stresses, such as loss of independence, social stigma, and travel limitations. They all have deep impact on employment and social relationships for CKD patients, in spite of before or after dialysis. To keep the stability of pre-dialysis, life quality of patients is a key to the smooth and successful treatment intervention and patient buy-in; therefore, this is a must-consider item for dialysis modalities. Meanwhile, the transfer of key information and knowledge and patient's decision-making 
are influenced by their emotions, including, fear and denial, such emotions came from inexperience and misunderstanding of their disease and what do they actually need during the treatment process, these are all negative influences when making subsequent decisions after receiving relevant information, thus the willingness of patients could be reduced. This happens to hold similar view and agrees with an earlier finding that emotions could interfere patients with information and background knowledge acquisition, and how they consider their dialysis experience. So the patients with fear of unknown consequences may lead them to perceive the choices as being more risky. To make more sensible and relatively correct decisions, screening known situations to identify bad emotions requires the intervention of various social work, and if necessary, the making of major treatment decisions should be postponed.

Based on a plenty of qualitative and quantitative studies and reports, family atmosphere and geographical location play a very important and decisive role in determining the therapeutic methods for ESRD patients. This is especially true in Iran and some Western countries where families have a high level of support for patients.[11, 12]. When choosing PD as therapeutic method, different regions and family atmosphere have both promoting and inhibiting effects on the patient's decision-making. This paper mainly discusses that different family backgrounds, such as good family interaction, structure, education level, ability of up-to-date knowledge acceptance, and expectation of life expectancy, may play a role in promoting the treatment of patients. While lower efficiency, economic ability and knowledge level of family members may inhibit the effect. In different studies, the role of families in the treatment process, in terms of supporting and helping them with getting better from disease, influenced their choice of therapeutic method [9]. In this study, we hope to conduct more investigations and studies on the influencing factors of patients, including the influences from themselves and their families, during the decision-making process of choosing treatment methods. We also believe that a more comprehensive understanding and analysis of these factors can lead to decisions that provide a better quality of life for patients.

In the future, if time and source of the guidance to patients could be tailored to their preferences and focus on their needs, it is possible that under such method, patient is highly expected to be more engaged into decision-making. For patients, adequate time and information can help them learn about which treatments are more appropriate to their own lifestyles, so they can be well prepared than willing to start dialysis. Only one optional curriculum cannot be suitable for all patients, they need more options. So we must come up with treatment plan one on one on the basis of patients' needs and requests. This means we need to develop a flexible CKD education curriculum and information resources library to tailor treatment models to patients, which requires patients to fully understand risks and benefits, and to deal with modality information in a practical way $[45,46]$. We often find that certain clinicians may have a limited ability to communicate risks and outcome probabilities with individualized patients, especially for those who are old. What is more, a clearer and more improved role clarification is also required, as patients frequently fail to identify their roles precisely. Studies $[34,36]$ have shown that classes should contain caregiver-focused information and coping skills. In general, medical staff should adopt a scientific method of education and guidance and fully respect the patient's right to participate in treatment decision-making.

In addition, sociocultural diversity requires us to take into account the diverse population of ESKD patients who have different ethnic, religious and socioeconomic backgrounds. In countries influenced by traditional Confucian culture, including China, the freedom of individual decision-making is relatively weak, and the family plays an extremely important role in it. Italian culture is dominated by the nuclear family, so patients' decisions are more influenced by the family. Especially in patients with religious beliefs, most of their views on the disease maintain a positive attitude. Elderly patients with religious beliefs tend to choose conservative treatment because of their personal beliefs.

\section{Conclusion}

This review aims to use a meta-synthesis approach [44] to synthesize thirty-two recently published qualitative studies. This research proposed what is worthy of further study and discussion in future qualitative and quantitative research, and suggested that the practice of doctor-patient SDM should be closely combined with the patient's belief, emotion and consciousness so as to improve the efficiency of intervention. Most of the studies used in this review focused on older patients, some of their perspectives have been reflected and presented on this paper. Further research is needed to understand the perspectives of family members on renal replacement therapy decisions.

Supplementary Information The online version contains supplementary material available at https://doi.org/10.1007/s11255-021-02913-8.

Authors' contributions YS and WL carried out the literature search and drafted the manuscript. SY, FJD, and SP were involved in the design of the study and performed the statistical analysis. HMP and MH assisted with the database searches and data extraction and examined the manuscript. YL conceived the study, participated in the study design and was instrumental in drafting the manuscript and critically reviewed the manuscript. All authors have read and approved the final manuscript.

Funding Nursing Innovation Fund of Chongqing Xinqiao Hospital (2019D088), People's Republic of China. Technology Innovation 
Projects for Social Undertakings and Livelihood Security in Chongqing Municipal Government (cstc2018jscx-msybX0056), People's Republic of China.

Availability of data and material PubMed, CINAHL, EMBASE, Cochrane Library, and China National Knowledge Infrastructure (CNKI) databases were used to identify all relevant published articles for review. These articles are open to the public.

Code availability Not applicable.

\section{Declarations}

Conflict of interest The authors have no interest conflicts regarding to this study.

Ethics approval and consent to participate In accordance with the ethical standards of institutional and international research committee, this article is based on previous published studies, thus it does not contain any studies with human participants or animals.

Open Access This article is licensed under a Creative Commons Attribution 4.0 International License, which permits use, sharing, adaptation, distribution and reproduction in any medium or format, as long as you give appropriate credit to the original author(s) and the source, provide a link to the Creative Commons licence, and indicate if changes were made. The images or other third party material in this article are included in the article's Creative Commons licence, unless indicated otherwise in a credit line to the material. If material is not included in the article's Creative Commons licence and your intended use is not permitted by statutory regulation or exceeds the permitted use, you will need to obtain permission directly from the copyright holder. To view a copy of this licence, visit http://creativecommons.org/licenses/by/4.0/.

\section{References}

1. Global, regional, and national burden of chronic kidney disease, 1990-2017: a systematic analysis for the Global Burden of Disease Study 2017 (2020). Lancet, 395(10225):709-733. https://doi. org/10.1016/s0140-6736(20)30045-3.

2. Cockwell P, Fisher LA (2020) The global burden of chronic kidney disease. Lancet 395(10225):662-664. https://doi.org/10.1016/ s0140-6736(19)32977-0

3. Glassock RJ, Warnock DG, Delanaye P (2017) The global burden of chronic kidney disease: estimates, variability and pitfalls. Nat Rev Nephrol 13(2):104-114. https://doi.org/10.1038/nrneph.2016. 163

4. Shay LA, Lafata JE (2015) Where is the evidence? A systematic review of shared decision making and patient outcomes. Med Decis Making 35(1):114-131. https://doi.org/10.1177/02729 $89 \times 14551638$

5. Vélez-Bermúdez M, Christensen AJ, Kinner EM, Roche AI, Fraer M (2019) Exploring the relationship between patient activation, treatment satisfaction, and decisional conflict in patients approaching end-stage renal disease. Ann Behav Med 53(9):816826. https://doi.org/10.1093/abm/kay091

6. Jayanti A, Neuvonen M, Wearden A, Morris J, Foden P, Brenchley $\mathrm{P}$ et al (2015) Healthcare decision-making in end stage renal disease-patient preferences and clinical correlates. BMC Nephrol 16:189. https://doi.org/10.1186/s12882-015-0180-8
7. Stacey D, Légaré F, Lewis K, Barry MJ, Bennett CL, Eden KB et al (2017) Decision aids for people facing health treatment or screening decisions. Cochrane Database Syst Rev 4(4):Cd001431. https://doi.org/10.1002/14651858.CD001431.pub5

8. Cassidy BP, Getchell LE, Harwood L, Hemmett J, Moist LM (2018) Barriers to education and shared decision making in the chronic kidney disease population: a narrative review. Can J Kidney Health Dis 5:2054358118803322. https://doi.org/10.1177/ 2054358118803322

9. Ozdemir S, Jafar TH, Choong LHL, Finkelstein EA (2019) Family dynamics in a multi-ethnic Asian society: comparison of elderly CKD patients and their family caregivers experience with medical decision making for managing end stage kidney disease. BMC Nephrol 20(1):73. https://doi.org/10.1186/s12882-019-1259-4

10. Fortnum D, Grennan K, Smolonogov T (2015) End-stage kidney disease patient evaluation of the Australian "My Kidneys, My Choice" decision aid. Clin Kidney J 8(4):469-475. https://doi. org/10.1093/ckj/sfv050

11. Hussain JA, Flemming K, Murtagh FE, Johnson MJ (2015) Patient and health care professional decision-making to commence and withdraw from renal dialysis: a systematic review of qualitative research. Clin J Am Soc Nephrol 10(7):1201-1215. https://doi.org/10.2215/cjn.11091114

12. Winterbottom AE, Gavaruzzi T, Mooney A, Wilkie M, Davies SJ, Crane D et al (2016) Patient acceptability of the yorkshire dialysis decision aid (YoDDA) booklet: a prospective non-randomized comparison study across 6 predialysis services. Perit Dial Int 36(4):374-381. https://doi.org/10.3747/pdi.2014.00274

13. Lockwood C, Munn Z, Porritt K (2015) Qualitative research synthesis: methodological guidance for systematic reviewers utilizing meta-aggregation. Int J Evid Based Healthc 13(3):179187. https://doi.org/10.1097/xeb.0000000000000062

14. Atkins S, Lewin S, Smith H, Engel M, Fretheim A, Volmink J (2008) Conducting a meta-ethnography of qualitative literature: lessons learnt. BMC Med Res Methodol 8:21. https://doi.org/ 10.1186/1471-2288-8-21

15. France EF, Ring N, Thomas R, Noyes J, Maxwell M, Jepson R (2014) A methodological systematic review of what's wrong with meta-ethnography reporting. BMC Med Res Methodol 14:119. https://doi.org/10.1186/1471-2288-14-119

16. Langlois EV, Tunçalp Ö, Norris SL, Askew I, Ghaffar A (2018) Qualitative evidence to improve guidelines and health decisionmaking. Bull World Health Organ 96(2):79-79a. https://doi.org/ 10.2471/blt.17.206540

17. Visser A, Dijkstra GJ, Kuiper D, de Jong PE, Franssen CF, Gansevoort RT et al (2009) Accepting or declining dialysis: considerations taken into account by elderly patients with endstage renal disease. J Nephrol 22(6):794-799

18. Murray MA, Thomas A, Wald R, Marticorena R, Donnelly S, Jeffs L (2016) Are you SURE about your vascular access? Exploring factors influencing vascular access decisions with chronic hemodialysis patients and their nurses. Cannt $\mathrm{j}$ 26(2):21-28

19. Harwood L, Clark AM (2014) Dialysis modality decision-making for older adults with chronic kidney disease. J Clin Nurs 23(2324):3378-3390. https://doi.org/10.1111/jocn.12582

20. Devitt J, Anderson K, Cunningham J, Preece C, Snelling P, Cass A (2017) Difficult conversations: Australian Indigenous patients' views on kidney transplantation. BMC Nephrol 18(1):310. https:// doi.org/10.1186/s12882-017-0726-Z

21. Cassidy BP, Harwood L, Getchell LE, Smith M, Sibbald SL, Moist LM (2018) Educational support around dialysis modality decision making in patients with chronic kidney disease: qualitative study. Can J Kidney Health Dis 5:2054358118803323. https:// doi.org/10.1177/2054358118803323 
22. Thomas N, Jenkins K, McManus B, Gracey B (2016) The Experience of older people in the shared decision-making process in advanced kidney care. Biomed Res Int 2016:7859725. https://doi. org/10.1155/2016/7859725

23. Rees J, Chilcot J, Donnellan W, Soulsby L (2018) Exploring the nature of illness perceptions in people with end-stage kidney disease. J Ren Care 44(1):19-29. https://doi.org/10.1111/jorc.12225

24. Lawrence C, Sharma S, Da Silva-Gane M, Fletcher BC, Farrington $\mathrm{K}$ (2013) Exploring the views of patients not on the transplant waiting list: a qualitative study. J Ren Care 39(2):118-124. https://doi.org/10.1111/j.1755-6686.2013.12012.x

25. Ahmadi SM, Jalali A, Jalali R (2018) Factors associated with the choice of peritoneal dialysis in iran: qualitative study. Open Access Maced J Med Sci 6(7):1253-1259. https://doi.org/10. 3889/oamjms.2018.255

26. Campbell-Crofts S, Stewart G (2018) How perceived feelings of "wellness" influence the decision-making of people with predialysis chronic kidney disease. J Clin Nurs 27(7-8):1561-1571. https://doi.org/10.1111/jocn.14220

27. Seah AS, Tan F, Srinivas S, Wu HY, Griva K (2015) Opting out of dialysis - Exploring patients' decisions to forego dialysis in favour of conservative non-dialytic management for end-stage renal disease. Health Expect 18(5):1018-1029. https://doi.org/ 10.1111/hex. 12075

28. Walker RC, Morton RL, Tong A, Marshall MR, Palmer S, Howard K (2015) Patient and caregiver preferences for home dialysis-the home first study: a protocol for qualitative interviews and discrete choice experiments. BMJ Open 5(4):e007405. https://doi.org/10. 1136/bmjopen-2014-007405

29. Dahlerus C, Quinn M, Messersmith E, Lachance L, Subramanian L, Perry E et al (2016) Patient perspectives on the choice of dialysis modality: results from the empowering patients on choices for renal replacement therapy (EPOCH-RRT) study. Am J Kidney Dis 68(6):901-910. https://doi.org/10.1053/j.ajkd.2016.05.010

30. Henry SL, Munoz-Plaza C, Garcia Delgadillo J, Mihara NK, Rutkowski MP (2017) Patient perspectives on the optimal start of renal replacement therapy. J Ren Care 43(3):143-155. https://doi. org/10.1111/jorc. 12202

31. Morton RL, Devitt J, Howard K, Anderson K, Snelling P, Cass A (2010) Patient views about treatment of stage 5 CKD: a qualitative analysis of semistructured interviews. Am J Kidney Dis 55(3):431-440. https://doi.org/10.1053/j.ajkd.2009.11.011

32. Erlang AS, Nielsen IH, Hansen HO, Finderup J (2015) Patients experiences of involvement in choice of dialysis mode. J Ren Care 41(4):260-267. https://doi.org/10.1111/jorc.12141

33. Gordon EJ (2001) Patients' decisions for treatment of end-stage renal disease and their implications for access to transplantation. Soc Sci Med 53(8):971-987. https://doi.org/10.1016/s02779536(00)00397-x

34. de Rosenroll A, Higuchi KS, Dutton KS, Murray MA, Stacey D (2013) Perspectives of significant others in dialysis modality decision-making: a qualitative study. Cannt j 23(4):17-24

35. Holvoet E, Verhaeghe S, Davies S, Combes G, François K, Johnson D et al (2020) Patients' experiences of transitioning between different renal replacement therapy modalities: A qualitative study. Perit Dial Int 40(6):548-555. https://doi.org/10.1177/08968 60819896219

36. Han E, Haldane V, Koh JJK, Quek RYC, Ozdemir S, Finkelstein EA et al (2019) Perspectives on decision making amongst older people with end-stage renal disease and caregivers in Singapore: a qualitative study. Health Expect 22(5):1100-1110. https://doi. org/10.1111/hex.12943
37. Balogun SA, May NB, Briley M, Bosch A, Duerr I, Owens JE et al (2019) A qualitative pilot study of the perceptions in older adults with end-stage kidney disease on hemodialysis. Can Geriatr J 22(2):55-63. https://doi.org/10.5770/cgj.22.342

38. Elliott BA, Gessert CE, Larson P, Russ TE (2012) Religious beliefs and practices in end-stage renal disease: implications for clinicians. J Pain Symptom Manage 44(3):400-409. https://doi. org/10.1016/j.jpainsymman.2011.09.019

39. King A, Lopez FY, Lissanu L, Robinson E, Almazan E, Metoyer $G$ et al (2020) Renal replacement knowledge and preferences for african americans with chronic kidney disease. J Ren Care 46(3):151-160. https://doi.org/10.1111/jorc.12312

40. Tweed AE, Ceaser K (2005) Renal replacement therapy choices for pre-dialysis renal patients. Br J Nurs 14(12):659-664. https:// doi.org/10.12968/bjon.2005.14.12.18287

41. DePasquale N, Ephraim PL, Ameling J, Lewis-Boyér L, Crews DC, Greer RC et al (2013) Selecting renal replacement therapies: what do African American and non-African American patients and their families think others should know? A mixed methods study . BMC Nephrol 14:9. https://doi.org/10.1186/ 1471-2369-14-9

42. Wong B, Venturato L, Oliver MJ, Quinn RR, Ravani P, HolroydLeduc J (2017) Selection of peritoneal dialysis among older eligible patients with end-stage renal disease. Nephrol Dial Transplant 32(2):384-392. https://doi.org/10.1093/ndt/gfw367

43. Finderup J, Jensen JD, Lomborg K (2020) Shared decision-making in dialysis choice has potential to improve self-management in people with kidney disease: A qualitative follow-up study. J Adv Nurs. https://doi.org/10.1111/jan.14726

44. Winterbottom A, Bekker HL, Conner M, Mooney A (2014) Choosing dialysis modality: decision making in a chronic illness context. Health Expect 17(5):710-723. https://doi.org/10.1111/j. 1369-7625.2012.00798.x

45. Lovell S, Walker RJ, Schollum JB, Marshall MR, McNoe BM, Derrett S (2017) To dialyse or delay: a qualitative study of older New Zealanders' perceptions and experiences of decision-making, with stage 5 chronic kidney disease. BMJ Open 7(3):e014781. https://doi.org/10.1136/bmjopen-2016-014781

46. Tonkin-Crine S, Okamoto I, Leydon GM, Murtagh FE, Farrington $\mathrm{K}$, Caskey $\mathrm{F}$ et al (2015) Understanding by older patients of dialysis and conservative management for chronic kidney failure. Am J Kidney Dis 65(3):443-450. https://doi.org/10.1053/j.ajkd.2014. 08.011

47. Subramanian L, Quinn M, Zhao J, Lachance L, Zee J, Tentori F (2017) Coping with kidney disease - qualitative findings from the Empowering Patients on Choices for Renal Replacement Therapy (EPOCH-RRT) study. BMC Nephrol 18(1). https://doi.org/10. 1186/s12882-017-0542-5

48. Boateng EA, East L, Evans C (2018) Decision-making experiences of patients with end-stage kidney disease (ESKD) regarding treatment in Ghana: a qualitative study. BMC Nephrol 19(1). https://doi.org/10.1186/s12882-018-1175-z

49. Loiselle MC, Michaud C, O'Connor A (2016) Decisional needs assessment to help patients with advanced chronic kidney disease make better dialysis choices. Nephrol Nurs J. 43(6):463-493

Publisher's Note Springer Nature remains neutral with regard to jurisdictional claims in published maps and institutional affiliations. 